\title{
3D Swing Trajectory of the Club Head from Depth Shadow
}

\author{
Seongmin Baek and Myunggyu Kim
}

\begin{abstract}
Using depth sensors like Microsoft Kinect for gesture recognition has recently drawn much academic attention. It is possible to recognize a user's body parts from depth images and find joint positions. Yet, it is not easy to track the club heads in fast swing motion. The present paper draws on single depth images to propose a new method of tracking the hand and club head paths in swing motion. Although the club head depth information cannot be directly gained from a depth sensor, a 3D trajectory can be generated by tracking and refining the hand and club head positions based on the depth-related shadow region information (depth shadow), hand depth information and club head depth information in the initial swing. The present experimental findings indicate the proposed method can easily capture the 3D hand and club head positions in driver tee shot motion in a small room.
\end{abstract}

Index Terms-Kinect, depth shadow, club head trajectory, depth image, hand position.

\section{INTRODUCTION}

The advent of the consumer depth camera (RGB-D) has triggered diverse research in the field of markerless gesture recognition, with a range of applications being released. As for games, cases of using users' gestures as interfaces such as experience sports and dance games are on the rise. In sports, gesture recognition is applied to posture coaching. Yet, it is hard to precisely recognize gestures with a single depth sensor unit due to self-occlusion.

Using multiple depth sensors minimizes invisible joint parts, solving the problem of joint occlusion, by restoring 3D gestures with camera calibration in combination with multi-joint data [1], [2]. As a method of estimating exact 3D postures from single depth images, the best matching pose is searched in a pre-captured motion database, and directly fitted to the depth value, so as to improve the initially estimated posture [3]. Shen et al. optimized the temporal motion consistency and the systematic bias to enhance the pose refinement and correction process [4]. However, the methods they suggested were intended to restore poses in line with the user's gestures but cannot be applicable to tools for sporting motions (e.g. golf clubs).

Analyzing golf swings using inertial sensors, S. Chun et al. attached inertial sensors onto the wrist and the grips on golf clubs to propose a golf coaching model capable of analyzing and assessing users' uncocking motion [5]. C. N. K. Nam et al. used inertial sensors and stereo cameras to propose a

Manuscript received August 10, 2015; revised January 15, 2016. This work was supported by Institute for Information \& communications Technology Promotion (IITP) grant funded by the Korea government (MSIP) (No. B0101-15-0152)

The authors are with Electronics and Telecommunications Research Institute, Daejeon, Korea (e-mail: baeksm@etri.re.kr,mgkim@etri.re.kr). method of tracking the movement of golf clubs in golf swing motion [6]. Still, sensors need be attached to body parts or clubs, which is inconvenient.

In a study intended to track a club's trajectory from 2D images, V. Lepetit et al. viewed data association as a matter of motion model fitting, and used a sliding window including a few frames around the current frame, instead of tracking frames one by one, to fit the target object's candidate position with a motion model, proposing a method of tracking the movement of a tennis ball or a golf club head [7]. N. Gehrig et al. applied a simple polar, polynomial approximation for modeling the club-head path, and generated the golf club's path by experimentally modeling ordinary upswings with the 4th polar curve and downswings with the 6th polar curve [8]. Yet, it is hard to track a fast moving golf club in 2D images due to blurring. Also, the final output is a 2D path at best.

As an inexpensive depth sensor, Microsoft Kinect takes a shot at 30fps maximum, and is not free from blurring images. This paper tracks the shadow region arising while capturing the depth images with Kinect to propose a new method of generating a 3D club head trajectory.

\section{Overview of Approach}

The present system uses the depth images (30fps@512×424) captured with a Kinect sensor unit. While a user is engaging in a swing motion, the body depth data can be captured without the fast moving club head depth data Thus, there is no yielding the club-head position directly. That is why the analysis of swings with a single Kinect unit yields nothing but user's body motion [9].

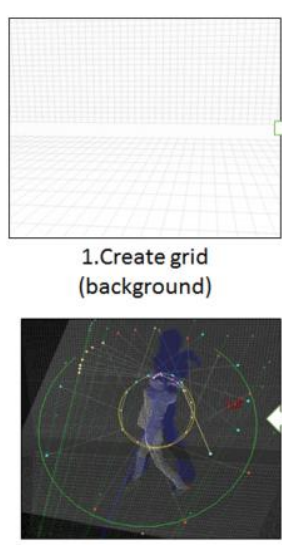

6.Curve fitting

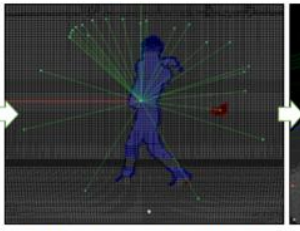

2.Shadow of club head tracking

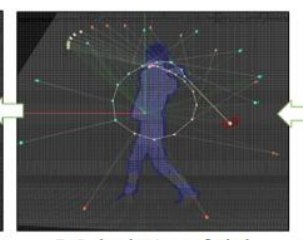

5.Calculation of club head position

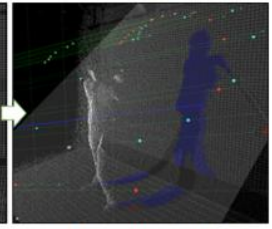

3.Projection plane for initial head position

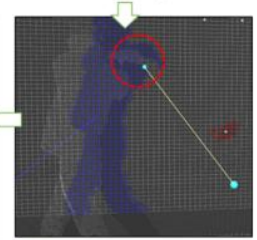

4. Hand point searching
Fig. 1. Basic processing pipeline of algorithm.

To find the shadow arising in depth images, the background depth images are gained first, and the grids are generated at the bottom ( $X Z$ plane) and the back side $(X Y$ plane). The number of depth points projected on the background and that of the depth points projected on the grid 
in the swing motion are compared with each other to locate the club-head shadow. Hand positions are estimated based on the $3 \mathrm{D}$ initial club head position on the virtual swing plane. Then, the 3D head position is calculated using the club length. The hand position is refined in relation to the club-head position and length. Finally, the spline curve is used to restore the $3 \mathrm{D}$ hand and club head trajectories.

The prerequisites for the system include the plane as flat as possible for capturing shadows and the wall behind the user. Fig. 1 illustrates the entire process of estimating the club-head and hand positions.

\section{BACKGROUND REGISTRATION}

With the captured depth images, the principal point and the focal length provided in Kinect SDK are applied as in the formula 1 and arranged in the 3D space (Fig. 2).

$$
\begin{aligned}
& W_{x y}=\left(I_{x y}-P_{x y}\right) \times D_{x y} / F_{x y} \\
& W_{z}=D_{x y}
\end{aligned}
$$

Here, $I$ is the value for a $2 \mathrm{D}$ image pixel. $P$ is the principal point. $F$ is the focal length. $D$ is the depth value. The points in the $3 \mathrm{D}$ space are represented as $W_{x y z}$.

Background registration is intended to generate the $\operatorname{Grid}\left(G_{X Z}, G_{X Y}\right)$ at the bottom (XZ plane) and the back side ( $X Y$ plane), and count the projected points. As noises frequently occur at the top and bottom as well as on the left and right sides of the depth images, the grid generation should be limited to the region that captures the user's movement including the club, which reduces the noises and shortens the calculation time.

The bottom grid generated is $N_{X} \times M_{Z}$, whilst the backside grid is $N_{X} \times M_{Y}$. Here, $N$ and $M$ are the number of grids and determined by the grid sizes. When the grid size is too big, the head's shadow region can be missing, whereas when the size is too small, the number of shadow regions to be searched increases due to noises. Here, the size of $4 \times 3$ is set to fit the head shadow size.

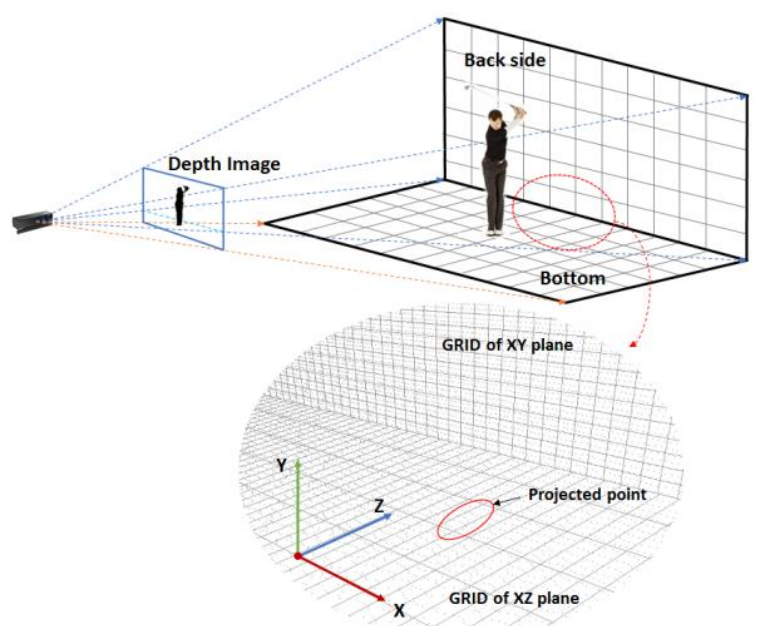

Fig. 2. Grid with projected points.

The position of the grid plane for projecting the 3D depth points is determined by the average height of the bottom and the average depth of the back side in the background.

Once the grid size and position are determined, the 3D points are projected onto each grid. Then, the number of projected points included within the grid $\left(N_{i j}, N_{i k}\right)$ need be counted.

The grid space including the points projected onto the bottom and the back side is shown in Fig. 2.

Finally, the golf ball position $\left(P_{b}\right)$ need be sought. As the ball is placed in a fixed position, it is possible to get its depth value, based on which its initial 3D position can be found with ease. The ball's initial position is used in chapter 4 to make a projection plane.

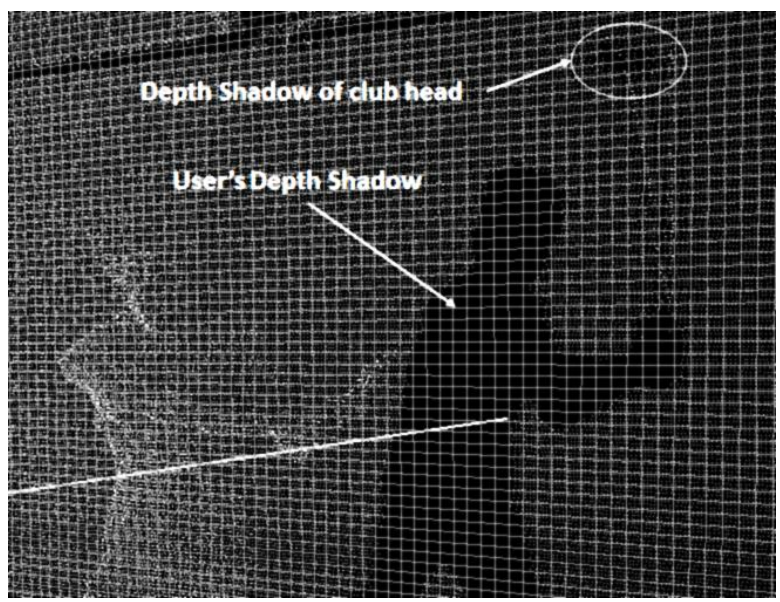

Fig. 3. Example of depth shadow.

\section{SHADOW SEARCH}

\section{A. Depth Shadow}

Kinect v2 emits light beam using TOF method and measures the duration of reflection to calculate the distance. Thus, when an object is fed into the sensor region, it reflects light, which causes the back side of the object to seemingly cast a shadow. When the other points, excluding the 3D depth points of the object, are projected onto the background grid, the grid corresponding to the shadow hardly shows points. This part is defined as the depth shadow (Fig. 3).

The club head moves so fast that depth values cannot be yielded in the swing motion. Yet, as in Fig. 3, the depth shadow occurs in the grid region. The depth shadow, $S(i, j)$, $S(i, k)$, is regarded to go lower than the number $\left(N_{i j}, N_{i k}\right)$ of points projected onto each grid that were pre-calculated in the background registration $\left(G_{X Z}, G_{X Y}\right)$.

$$
\begin{array}{rlrl}
S(i, j) & =\text { true, } & & \text { if } \operatorname{Count}\left(G_{N_{i j}}<N_{i j} \times \alpha\right) \\
& =\text { false, } & \text { else }
\end{array}
$$

Here, $\alpha=0.5$ and $G_{N i j}$ is the number of points within grid.

\section{B. Depth Shadow of User}

It is the user that takes up the largest part of the depth shadow region in the grid. As the purpose here is to find the head shadow, the shadow region caused by the user is excluded.

As the user stands at the center of the Kinect sensor, first, it is necessary to verify whether the grid $\left(G_{X C Y C}\right)$ corresponding to the center in the backside grid $\left(G_{X Y}\right)$ is in the shadow region. If it is, it should be set to 1 , and fed into the shadow region queue. The center grid serves as the starting point for searching the user's shadow region. Eight grids adjacent to the starting grid need be determined. If each grid is in the 
shadow region, it should be set to 1 and fed into the shadow region queue. Each of the grids fed into the shadow region queue need be taken out, in order to double check if the adjacent grids not set to 1 are in the shadow region and save them. This process need be repeated until the shadow region queue becomes empty.

As for the starting point of the bottom grid $\left(G_{X Z}\right)$, the region that is identical to the grid's X-axis index value at the point of meeting the back-side grid is fed into the queue as the starting point $\left(G_{X k Z m-1} \leftarrow G_{X k Y o}=1\right)$. Following the process aforementioned, adjacent grids need be verified.

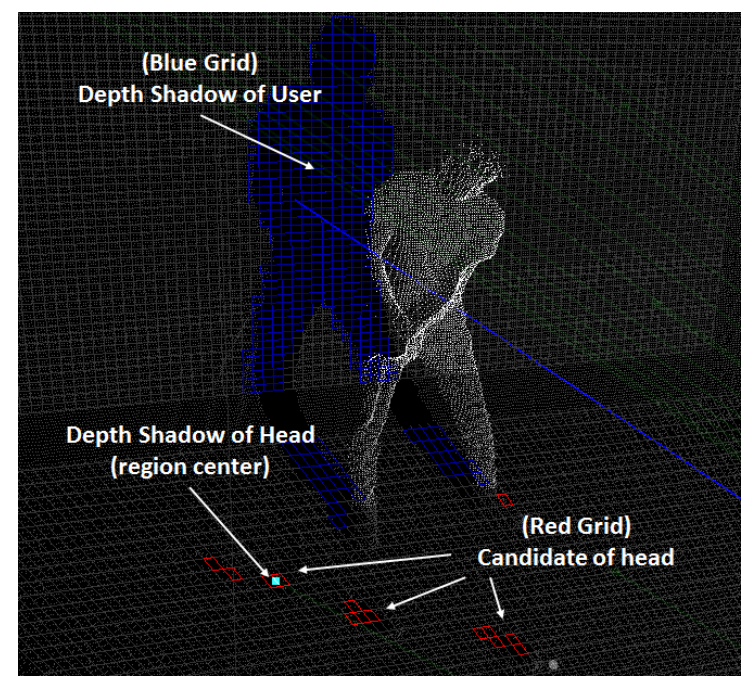

Fig. 4. Shadow region of user and club head.

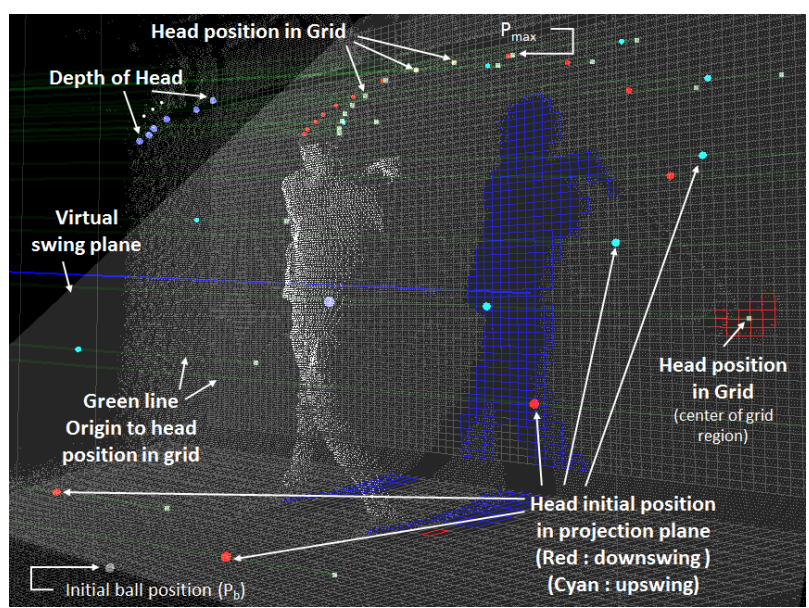

Fig. 5. Virtual swing plane $\&$ initial $3 \mathrm{~d}$ club head position.

Upon completion of the process, the shadow region caused by the user is extracted. From the grid region excluding the shadow region caused by the user $\left(G_{i j} \neq 1\right)$, the shadow cast by the club head is found. The following pseudo code shows the process of searching the user's depth shadow.

$$
\begin{aligned}
& \text { ShadowQueue.Add }\left(G_{X_{C} Y_{C}}\right) \text {, if }\left(G_{X_{C} Y_{C}}=1\right) \\
& \text { for } G_{c u r} \text { in ShadowQueue } \\
& \text { for } G_{\text {neighbor }} \text { of } G_{\text {cur }}, G_{\text {neighbor }} \neq 1 \\
& \text { if } G_{\text {neighbor }}==\text { depth shadow } \\
& G_{\text {neighbor }}=1 \\
& \text { ShadowQueue.Add }\left(G_{\text {neighbor }}\right)
\end{aligned}
$$

\section{Depth Shadow of Club Head}

The depth map generated by Kinect may be mistaken for the shadow region as the number included in the grid varies with noises. Also, due to the fast swing speed of the club, blurring occurs even in the shadow region, which makes it hard to determine the exact position. In addition, the club shaft may cast a shadow. Here, the purpose is to track the parts corresponding to the head in several shadow regions attributable to noises and other factors.

Following the above mentioned method, the depth shadow should be found in the grid excluding the user region. The shadow region found is subject to segmentation to generate a block consisting of adjacent grids. If a single block is generated, it is considered the head region. Yet, if more than two blocks are formed, the head region should be found as follows:

- A block that takes up a significant number of grids in comparison to other blocks; and

- A block closer to the position predicted based on the previous head shadow position and velocity.

When the initial swing is about to start, the head speed is 0 . Subsequently, up to 2 3 frames, the speed is not so fast that a large shadow region cast by the head can be captured, making it easy to find the head shadow region. Then, the current position can be determined based on the previous position and velocity.

Here, it is necessary to find out if the head's depth data is on the line that connects the starting point in the coordinate system with the head shadow region's central point. At the very moment that the initial swing starts, or for a few frames when the swing starts to move from the halt state, the head's depth data can be obtained.

The obtained head depth data is used to correct the occluded hand position as well as the head's 3D position.

\section{3D TRAJECTORY}

Based on the club head's shadow region, this paper proposes a straightforward method of tracking the 3D positions of the club head and the hand.

The 3D head and hand positions are found as follows. First, based on the club-head shadow region, a virtual swing plane need be generated. Then, the club-head position should be moved onto the virtual swing plane. The depth points close to the estimated initial club-head position are found. The selected depth points are assumed as the endpoint position of the hand, from which the actual 3D club-head position is sought. The hand position, which is inaccurate due to occlusion, can be corrected based on the club-head position. Details of each process are described below.

\section{A. Virtual Swing Plane \& Initial Club Head Position}

A shadow cast in the background implies that the $3 \mathrm{D}$ head position is present on the line connecting the starting point in the camera's coordinate system with the club head's shadow region. Still, it is not possible to establish the exact point on the line as the head's position. Thus, additional information is needed. To that end, the user's hand position is estimated Still, selecting the points closest to the head's shadow region may lead to mapping other parts, not the hand.

To track the exact hand position, a virtual swing plane is defined here, given that the golf swing motion moves along the downswing plane. 
To generate the projection plane, the ball's initial position $\left(P_{b}\right)$ and the club head shadow region of the highest y value are used (Fig. 4).

$$
\begin{aligned}
& V_{S}=\left(P_{\max }+C_{Z}\right)-P_{h} \\
& V_{X}=(1,0,0) \\
& N_{P}=\operatorname{Normal}\left(V_{S} \times V_{X}\right)
\end{aligned}
$$

Here, $P_{\max }$ is the position of the highest y value in the head shadow region. $\boldsymbol{C}_{Z}$ vector used to correct the depth value is a value for approaching the range of the club's movement. The projection plane's normal vector is $N_{p}$.

The point where the line that connects the starting point in the camera's coordinate system with each head shadow region meets the projection plane is set as the initial head position $\left(i H E_{t}\right)$. Fig. 5 shows the generated virtual swing plane and the initial head position.

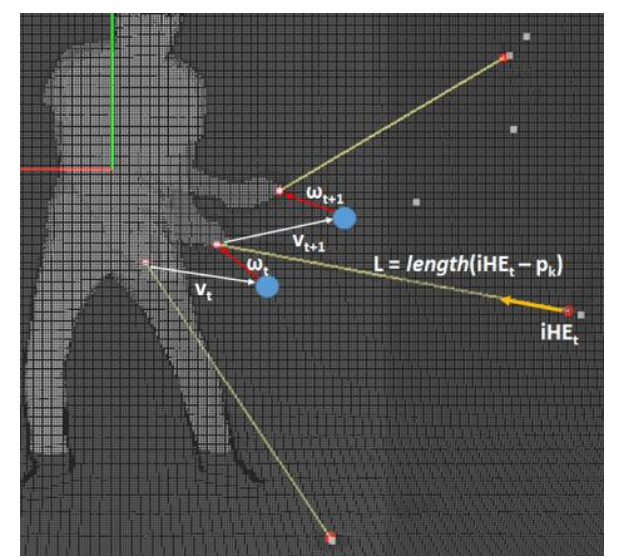

(a) Hand point selection by initial head position and previous hand velocity

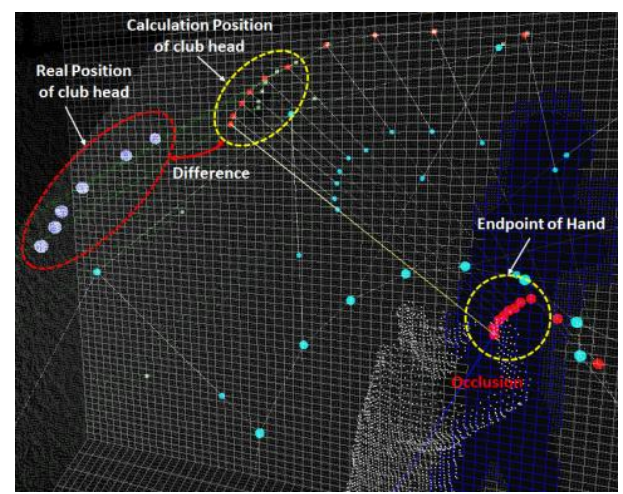

(b) Wrong point selection of hand by occlusion and the difference between real depth point and calculation point of club head

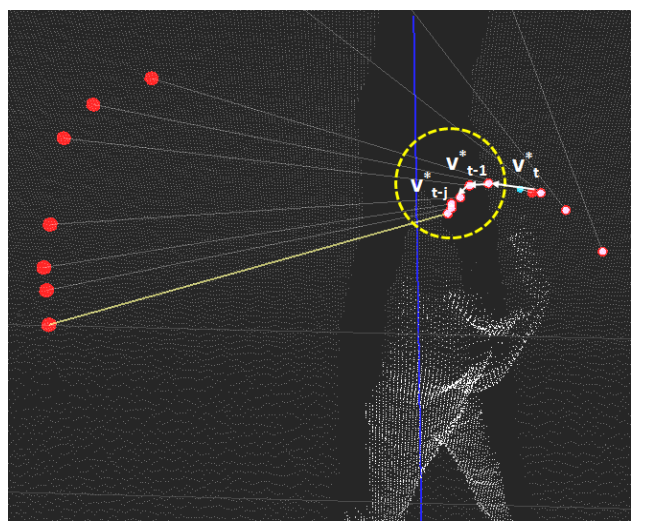

(c) Hand position correction with hand velocity and club head depth data Fig. 6. Hand position in the downswing.

The projection plane is intended for approximation to the downswing plane so as to estimate the $3 \mathrm{D}$ position of the user's hand based on the initial club head position. Thus, if not an exact head position, the hand position can be estimated more accurately than seeking its position directly in the head shadow region.

\section{B. 3D Hand Position}

As the depth information is gained from a single depth sensor, the hand parts are occluded depending on the swing time, which makes it difficult to find the endpoint position of the hand grabbing the club. Hence, the point when the hand position is most visible in the swing, or the point when the head shadow position occurs on the $X Z$ plane, is used as the starting point for tracking the hand.

As the hand comes forward when the club is about to hit the ball, a point closest to the initial club head position $\left(i H E_{t}\right)$ is selected as the initial hand position $\left(i H A_{t}\right)$. Based on the hand position and velocity calculated in the current frame $(\mathrm{t}$ frame), the previous frame $(t-1)$ is used to track the hand position in the downswing, whilst the next frame $(t+1)$ is used to track the hand position in the upswing.

$$
\begin{aligned}
& l_{t \pm 1}=\left|i H E_{t \pm 1}-P_{k}\right| \times \omega_{t} \\
& \omega_{t}=\left|\left(i H A_{t}+V h_{t}\right)-P_{k}\right| \\
& i H A_{t \pm 1}=P_{k} \text { with shortest }\left(l_{t \pm 1}\right)
\end{aligned}
$$

Here, $t+1$ and $t-1$ refer to the upswing and the downswing directions, respectively. $P_{k}(k=1 \ldots n)$ is the $3 \mathrm{D}$ user depth point. $\omega_{t}$ is the weighted value, which is defined as the distance between the position in line with the hand velocity and the points.

Despite being straightforward, the proposed method proves to successfully track the endpoint position of the hand in the experiment (Fig. 6(a)).

However, when the hand moves backward, it is not possible to find the exact position of the hand due to occlusion (Fig. 6(b)). If the hand position shows an insignificant difference from the previous value, or if it moves in a direction different from the expected one, the estimated value is set as the initial hand position (Fig. 6(c)).

\section{3D Head Position}

Based on the initial hand position tracked in every frame, the $3 \mathrm{D}$ club-head position is tracked. It is assumed that the club length, $L$, is already known. As above mentioned, the 3D head position is present on the line that connects the starting point in the coordinate system with the head shadow region. Thus, it becomes a matter of finding the position on the line at a distance of $L$ from the initial hand position, which is easily calculated with the second law of cosines.

$$
\begin{aligned}
& T^{2}=\overrightarrow{H A}^{2}+L^{2}-2 \overrightarrow{H A} \cdot L \cdot \cos (\theta) \\
& H E=\overrightarrow{O S_{C}} \cdot( \pm T)
\end{aligned}
$$

Here, $O S_{c}$ is the vector that connects the starting point $(O)$ in the camera's coordinate system with each head shadow center $\left(S_{c}\right)$.

As a rule, two solutions exist. Here, based on the previous $3 \mathrm{D}$ club head position and velocity, the closer point is chosen.

When the swing starts, the club's depth value can be yielded within the first 2 3 frames. This value becomes the actual 3D head position and serves as the reference point for 
the head position and velocity.

\section{D. $3 D$ Hand Position Refinement}

Once the $3 \mathrm{D}$ club head position is determined, the club length, $L$, is used again for the refinement of the initial hand position because there may be a gap between the position at a distance of $L$ from the 3D head position toward the hand, and the hand position. In particular, when the hand is occluded by the head, the exact hand position is hard to find, leading to a significant gap.

If the gap exceeds the threshold, the hand position is refined to the position at a distance of $L$ from the $3 \mathrm{D}$ club-head position, which benefits the calculation of the hand path. Notably, as the actual club-head depth data can be gained in a couple of first frames, the hand position can be refined based on such frames, which ensures a better calculation.

Once the hand and club-head positions are yielded in every frame, the hand and head trajectories are calculated based on the curve fitting. Fig. 7 and Fig. 8 shows the 3D position and trajectory results for the hand and the head, respectively.

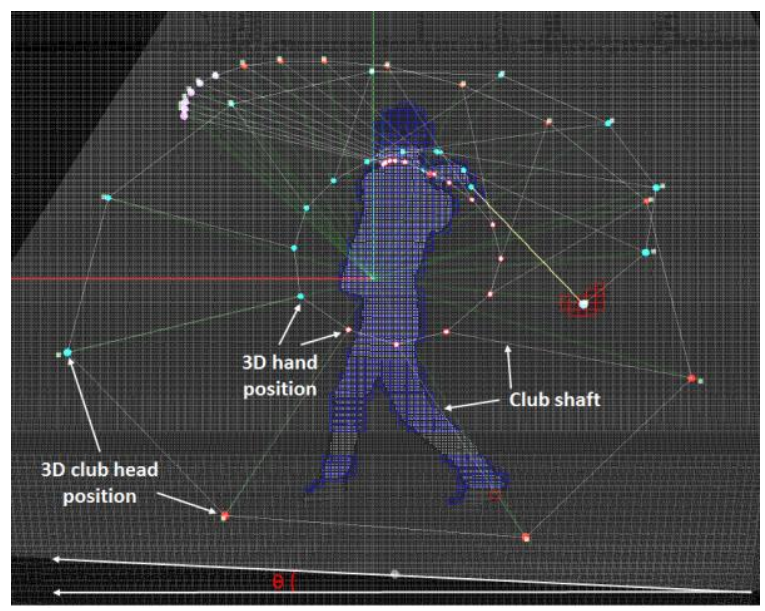

Fig. 7. Hand \& club head position in the downswing (red) and upswing (cyan).

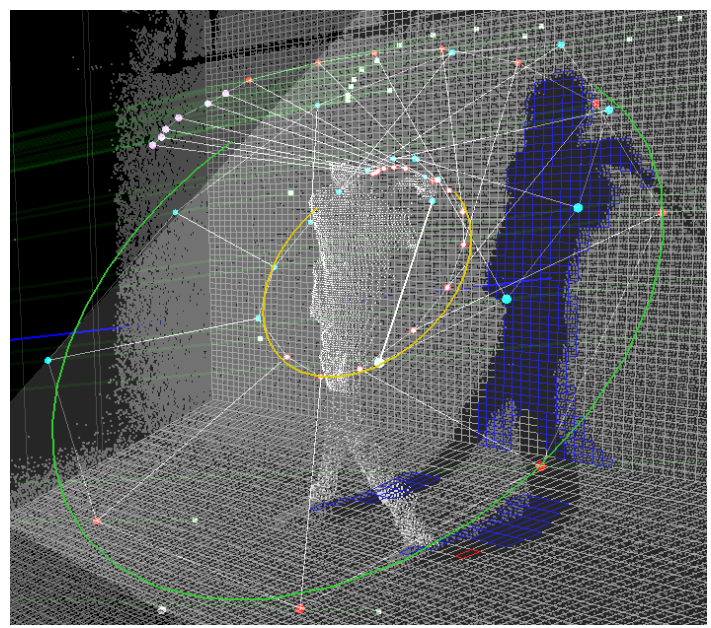

Fig. 8. 3D trajectories of hand (yellow) \& club head(green).

\section{CONCLUSION}

The golf club used for the experiment is a driver. A professional golfer's driver swing speed is $150 \mathrm{MPH}$ on average, while an amateur's is $75 \sim 100 \mathrm{MPH}$ on average. As

the Kinect sensor collecting information operates at 30fps, just a few shots of depth images are captured in the fast swing. Also, as the shutter speed cannot be controlled, much blurring occurs. In addition, as the fast moving club head's depth information cannot be captured even with the depth sensor, it is tricky to restore the $3 \mathrm{D}$ club head trajectories.

The present paper draws upon the depth images fed into a single unit of Kinect sensor to propose a new method of tracking the club head trajectories based on depth shadows. Unlike the established images showing nothing but the user's posture, or the $2 \mathrm{D}$ trajectories tracked in $2 \mathrm{D}$ images, the proposed method can yield 3D club head and hand trajectories at the same time, which facilitates coaching the swing motion.

In future works, sensors will be attached to the club and hands to track actual positions, which will be compared with the $3 \mathrm{D}$ positions calculated with the proposed method, with a view to quantifying the accuracy. A more accurate and precise 3D tracking will be possible by analyzing and using the gaps in distance for refinement.

\section{REFERENCES}

[1] A. Naveed, "Unified skeletal animation reconstruction with multiple kinects," in Proc. Eurographics (Short Papers), 2014, pp. 5-8.

[2] S. Baek and M. Kim, "Dance experience system using multiple kinects," Int. Journal of Future Computer and Communication, vol. 4 , pp. 45-49, 2015.

[3] M. Ye, X. Wang, R. Yang, L. Ren, and M. Pollefeys, "Accurate 3-D pose estimation from A single depth image," in Proc. ICCV, 2011, pp. 731-738.

[4] W. Shen, K. Deng, X. Bai, T. Leyvand, B. Guo, and Z. Tu, "Exemplar-based human action pose correction and tagging," in Proc. IEEE Conf. CVPR, pp. 1784-1791, 2012.

[5] S. Chun, D. Kang, H. R. Choi, A. Park, K. K. Lee, and J. Kim, “A sensor-aided self coaching model for uncocking improvement in golf swing," Multimedia Tools Appl., vol. 72, pp. 253-279, Sep. 2013.

[6] C. N. K. Nam, H. J. Kang, and Y. S. Suh, "Golf swing motion tracking using inertial sensors and a stereo camera," IEEE Trans. Instrum. Meas., pp. 943-952, 2013.

[7] V. Lepetit, A. Shahrokni, and P. Fua, "Robust data association for online applications," in Proc. IEEE Conf. CVPR, 2003, vol. 1, pp. 281-288.

[8] N. Gehrig, V. Lepetit, and P. Fua, "Golf club visual tracking for enhanced swing analysis tools," in Proc. Brithsh Machine Vision Conference, Sep. 2003, pp. 1-10.

[9] Swinguru. [Online]. Available: http://swinguru.com
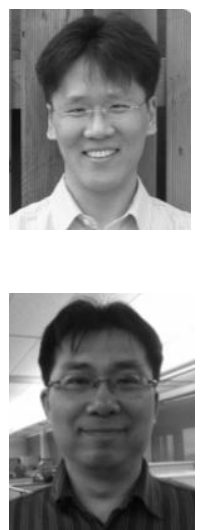

Seongmin Baek was born in 1973 in Korea. He received the MS degree in computer science (virtual reality) from Pohang University of Science and Technology (POSTECH), Korea in 2001.

$\mathrm{He}$ is working as a senior member of engineering staff at Visual Contents Research Department of ETRI with interest in digital contents, animation, and physics.

Myunggyu Kim was born in 1967 in Korea. He received the Ph.D. from University of Maryland at College Park (Department of Physics). He is working as a principal research scientist at Visual Contents Research Department of ETRI with interest in network, simulation, and physics. 